\title{
Acral pityriasis rubra pilaris
}

\author{
Michael Sander DMD, Megan Sander MD
}

Cite as: CMAJ 2020 February 3;192:E118. doi: 10.1503/cmaj.190961
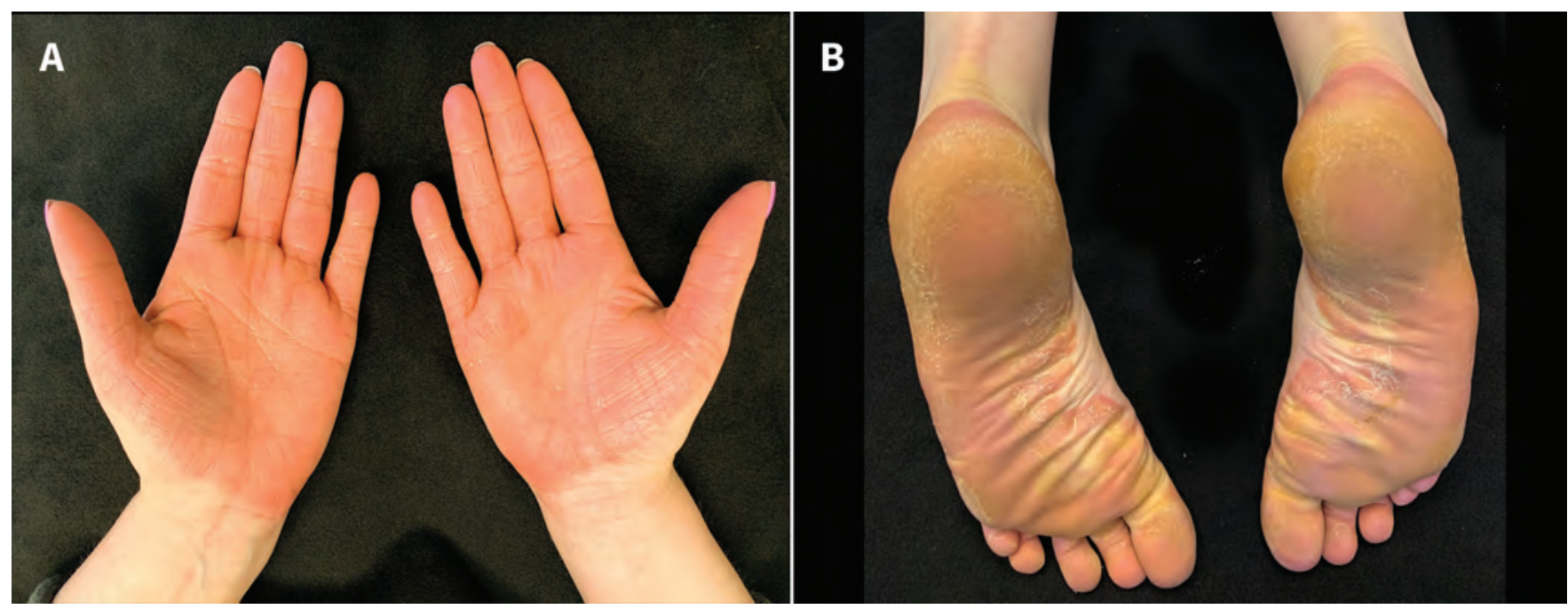

Figure 1: (A) Confluent, red-orange, waxy plaques on the palmar aspects of the hands of a 34-year-old woman with pityriasis rubra pilaris. (B) Similar red-orange plaques on the plantar aspects of the feet.

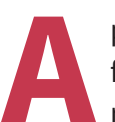

previously healthy 34-year-old woman presented to her family physician with an asymptomatic erythematous rash on her hands that had been present for several weeks. She had elevated levels of alanine transaminase, alkaline phosphatase and $\gamma$-glutamyltransferase, compatible with a diagnosis of primary biliary cholangitis. Because the morphology of the lesions was atypical, the patient was referred to a dermatologist.

There were well-circumscribed, red-orange, waxy plaques on the patient's palms and soles, compatible with a diagnosis of pityriasis rubra pilaris (Figures $1 \mathrm{~A}$ and $1 \mathrm{~B}$ ). Because of the plaques, it was unlikely to be palmar erythema secondary to hepatic disease, although psoriasis was a possibility. Results for HIV testing were negative. A biopsy supported the clinical impression of pityriasis rubra pilaris (Appendix 1, available at www.cmaj.ca/lookup/ suppl/doi:10.1503/cmaj.190961/-/DC1).

Pityriasis rubra pilaris is a cutaneous disease of unknown cause. It can be classified into 6 subtypes based on morphology, age of onset and HIV status, ${ }^{1}$ and although the prognosis of pityriasis rubra pilaris varies with subtype, it typically resolves within 3-5 years. ${ }^{1}$ Waxy keratoderma is a distinctive feature of all types of pityriasis rubra pilaris ${ }^{1}$ presenting with acral involvement, the texture being likened to carnauba wax. Waxiness is not a feature of non-acral pityriasis rubra pilaris. ${ }^{1}$ The association with primary biliary cholangitis is uncommon; therefore, the significance of the presence of primary biliary cholangitis in this patient is uncertain. ${ }^{2}$
Investigations should include a biopsy and testing for levels of liver enzymes and HIV serology. Acitretin is the first-line treatment, but alternative treatments include topical steroids, phototherapy, methotrexate and tumour necrosis factor- $\alpha$ inhibitors. ${ }^{3}$

The patient's pityriasis rubra pilaris was treated with topical steroids, as systemic treatment was contraindicated, together with ursodiol for her primary biliary cholangitis. At a 4-week follow-up, her plaques had improved, thinning by about $50 \%$.

\section{References}

1. Klein A, Landthaler M, Karrer S. Pityriasis rubra pilaris. Am J Clin Dermatol 2010;11:157-70.

2. Nadhan KS, Warner CG, van den Berg-Wolf M, et al. Palmoplantar keratoderma as a presenting sign of primary biliary cirrhosis. JAAD Case Rep 2017;4:41-3.

3. Wang D, Chong V, Chong W, et al. A review on pityriasis rubra pilaris. Am J Clin Dermatol 2018;19:377-90.

\section{Competing interests: None declared.}

This article has been peer reviewed.

The authors have obtained patient consent.

Affiliation: Department of Medicine, Cumming School of Medicine, University of Calgary, Calgary, Alta.

Acknowledgement: We wish to thank Dr. Amy Thommasen for the histopathology image as well as her expert opinion.

Correspondence to: Megan Sander, megan.a.sander@gmail.com 